\title{
Barriers and opportunities related to extracorporeal cardiopulmonary resuscitation for out-of-hospital cardiac arrest in Canada: A report from the first meeting of the Canadian ECPR Research Working Group
}

Steven C. Brooks, MD, MHSc ${ }^{*+}$; Sam D. Shemie, MD ${ }^{\text {***; }}$ Sylvia Torrance**; Laura Hornby, MSc ${ }^{* *^{+\dagger}}$; Clay Gillrie, MSN**; Brian Grunau, MD, MHSc ${ }^{\ddagger \neq \uparrow \pi} ;$ A. Dave Nagpal, MD***+t+; Andrew Baker, MD ${ }^{\neq \neq \neq \neq \S \S \S ; ~}$ Jim Christenson, $\mathrm{MD}^{\ddagger \neq \uparrow \pi}$; John Gill, MD, MSc ${ }^{\S \S}$; Laurie Morrison, MD, MSc ${ }^{\text {t§; }}$; on behalf of the Canadian ECPR Research Working Group

For a French translation of the executive summary, please see the Supplementary Material at DOI: 10.1017/cem.2017.429

\section{EXECUTIVE SUMMARY}

Extracorporeal cardiopulmonary resuscitation (ECPR) is an emerging therapy for patients with cardiac arrest refractory to conventional resuscitation. ECPR entails the use of extracorporeal membrane oxygenation (ECMO) to sustain perfusion in patients with cardiac arrest. Best available evidence from around the world is of low quality, but observational studies have suggested that ECPR is associated with increased survival in subgroups of out-of-hospital cardiac arrest (OHCA) patients and provides organ donation opportunities in non-survivors. Organized by Canadian Blood Services in collaboration with the Canadian Resuscitation Outcomes Consortium, we assembled an interdisciplinary group of clinicians and researchers from across Canada, with support from international experts, to discuss opportunities and barriers related to the use of ECPR for OHCA in the Canadian context. Representatives included those with expertise in prehospital medicine, emergency medicine, resuscitation, cardiac surgery, ECMO, neurology, neurointensive care, critical care, organ donation, transplantation, health policy, health economics, and bioethics.

There was a consensus from this interdisciplinary group that ECPR is a potentially viable strategy to improve survival for patients who suffer OHCA in Canada. The group agreed that further investigation is warranted because the evidence supporting this practice is not definitive, and equipoise remains. Critical elements of the patient journey from the prehospital setting to the emergency department and then ultimately to a critical care setting were defined by the working group and then discussed. The group agreed that the efficacy and cost-effectiveness of ECPR for OHCA in the Canadian setting need to be determined before considering broad implementation. In addition, the group identified several other high priority questions about the implementation

From the *Department of Emergency Medicine, Queen's University, Kingston, ON; †Rescu, Li Ka Shing Knowledge Institute, St. Michael's Hospital, University of Toronto, Toronto, ON; ¥Departments of Anesthesia and Surgery, Interdepartmental Division of Critical Care Medicine and; $\S$ Division of Emergency Medicine, Department of Medicine, University of Toronto, Toronto, ON; IDivision of Critical Care, Montreal Children's Hospital, McGill University, Montreal, QC; ${ }^{* *}$ Canadian Blood Services, Ottawa, ON; ††Division of Pediatric Critical Care, Children's Hospital of Eastern Ontario Research Institute, Ottawa, ON; ¥¥Department of Emergency Medicine and; §§Division of Nephrology, Department of Medicine, Faculty of Medicine, University of British Columbia, Vancouver, BC; ППSt. Paul's Hospital, Vancouver, BC; ***Division of Cardiac Surgery and;

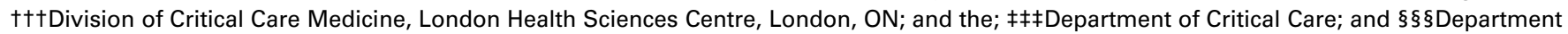
of Anesthesia, Keenan Research Centre for Biomedical Science, St. Michael's Hospital, Toronto, ON.

Correspondence to: Steven C. Brooks, Kingston General Hospital, Watkins 4, 76 Stuart Street, Kingston, ON K7L 2V7; Email: brookss1 @kgh.kari. net 
of ECPR for OHCA, including (but not limited to) the following:

1) Does a strategy involving ECPR, compared to optimal conventional resuscitation approaches of the same duration, improve clinical outcomes for patients with OHCA?

2) What are the best practices in ECPR to optimize neurologically favorable survival?

3) What are the requirements for system preparedness, capacity, training, and logistics related to an ECPR program?

4) How can prognostication be accurately done for patients treated with ECPR?

5) What is the optimal approach to end-of-life care for patients treated with ECPR?

6) What is the best strategy for optimizing organ donation opportunities for patients treated with ECPR who do not survive?

Opportunities for research and development in this field were identified with an emphasis on the need for collaborative interdisciplinary research on the efficacy, effectiveness, and feasibility of ECPR for OHCA in Canada. Future work should include the development and evolution of this working group into a national research collaborative, surveillance of the literature for data from ECPR clinical trials currently underway to guide our research agenda, the development of a minimum data set for ECPR research in Canada, and the development of pilot studies to support future clinical trial implementation.

\section{BACKGROUND}

Most patients with OHCA refractory to initial conventional cardiopulmonary resuscitation (CPR) and advanced life support measures will die. Treatment options with proven efficacy for this population are not available. ECMO is a form of heart-lung bypass that oxygenates and circulates blood externally to the body through cannulation of large arteries and veins. ECMO is used in some major hospital centres for a variety of indications, including respiratory failure, ${ }^{1}$ cardiac failure, ${ }^{2}$ septic shock, ${ }^{3}$ and, in some cases, refractory cardiac arrest. ${ }^{4}$ The use of ECMO for patients in refractory cardiac arrest, termed extracorporeal cardiopulmonary resuscitation, has emerged as a novel, yet unproven treatment option for refractory cardiac arrest. ECPR can be used to sustain life during cardiac arrest while the underlying cause is rectified. This may involve treatments such as primary percutaneous coronary intervention, dialysis of a toxin, temperature correction after hypothermia, or clot removal after pulmonary embolism.

\section{Purpose and objectives of the meeting}

We planned this meeting as the first step towards the development of a collaborative, multidisciplinary research consortium and a coordinated national research agenda to study ECPR for OHCA in Canada. The specific objectives were 1) to review the current knowledge and guidelines on the use of ECPR, 2) to delineate the clinical process related to ECPR for OHCA patients, 3) to identify knowledge gaps as they relate to the clinical process of ECPR, and 4) to identify logistic, ethical, and economic challenges associated with ECPR implementation and research in Canada.

\section{METHODS}

\section{Participant selection}

We selected 28 people to attend this meeting (Supplementary Online Appendix 1). Participants were selected by the planning committee based on expertise in ECMO, ECPR, a relevant clinical area, health economics, or bioethics. We used a snowball sampling method where invited participants had the opportunity to identify other potential participants. Areas of expertise among those attending included paramedicine, resuscitation science, emergency medicine, critical care, neurocritical care, cardiac surgery, organ donation, transplantation, ECMO, ECPR, bioethics, and health economics.

\section{Meeting process}

An inaugural meeting was held in Toronto on May 4, 2016 at the $\mathrm{Li} \mathrm{Ka}$ Shing Knowledge Institute of St. Michael's. Prior to the meeting, attendees were provided with a bibliography of selected ECPR studies to serve as background (see the reference list). The meeting was opened with presentations from invited national and international experts to provide context for the planned discussions. (See Supplemental Online Appendix 2 for the meeting agenda). Each presentation was followed by a large group discussion to probe the knowledge of 

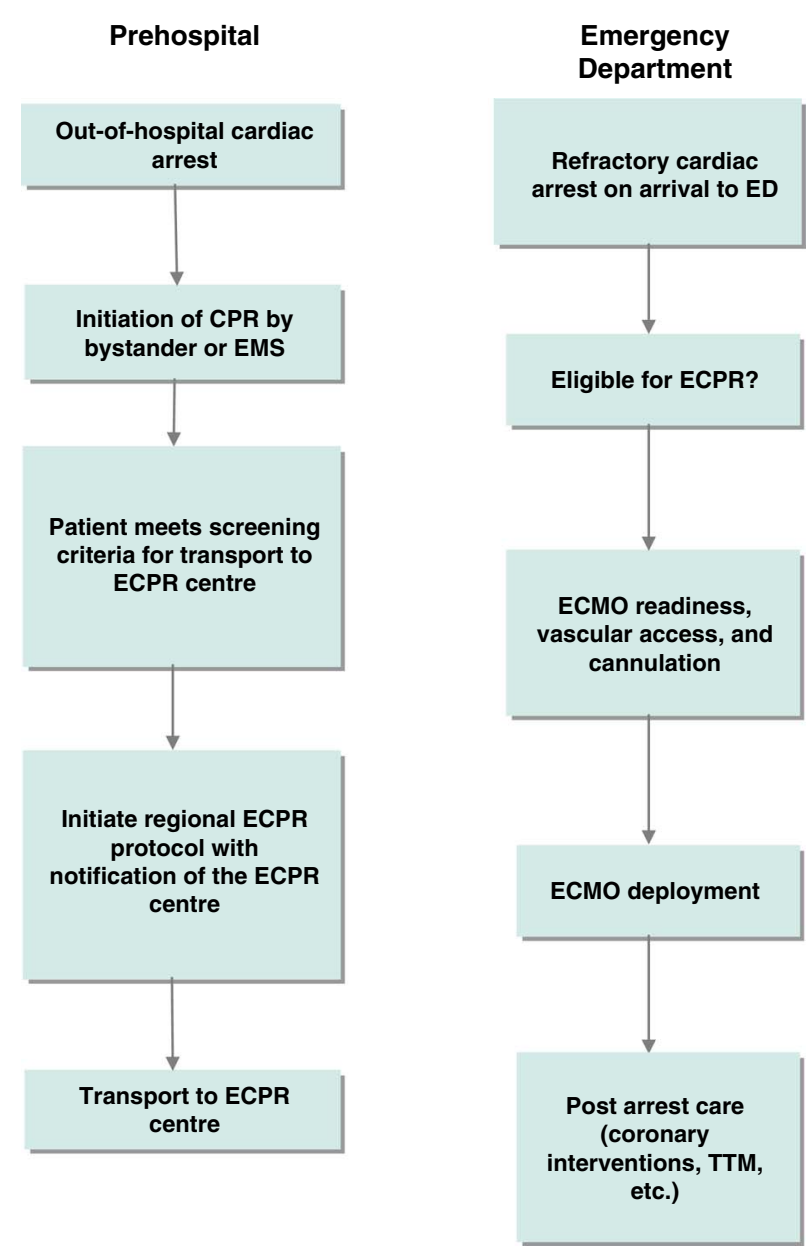

Figure 1. Clinical process frameworks for extracorporeal cardiopulmonary resuscitation in the prehospital and emergency department phases of care.

$\mathrm{CPR}=$ cardiopulmonary resuscitation;

$\mathrm{ECMO}=$ extracorporeal membrane oxygenation;

$\mathrm{ECPR}=$ extracorporeal cardiopulmonary resuscitation;

$\mathrm{ED}=$ emergency department; $\mathrm{EMS}=$ emergency medical

services; TTM = targeted temperature management.

presenters and consider the content of their presentations in the Canadian context. In the second part of the meeting, participants were organized into three smaller groups based on three phases of clinical care in the ECPR process (Figures 1 and 2). Each group was tasked with identifying high priority knowledge gaps to guide future research. Attendees were guided to determine priority by assessing the relationship of the knowledge gap to potentially improving patient outcomes and relevance to supporting the clinical implementation of ECPR for OHCA in the Canadian setting. Groups were provided with a flow diagram proposed by the meeting planning committee to serve as a framework for discussion (see Figures 1 and 2). A plenary session was held following the

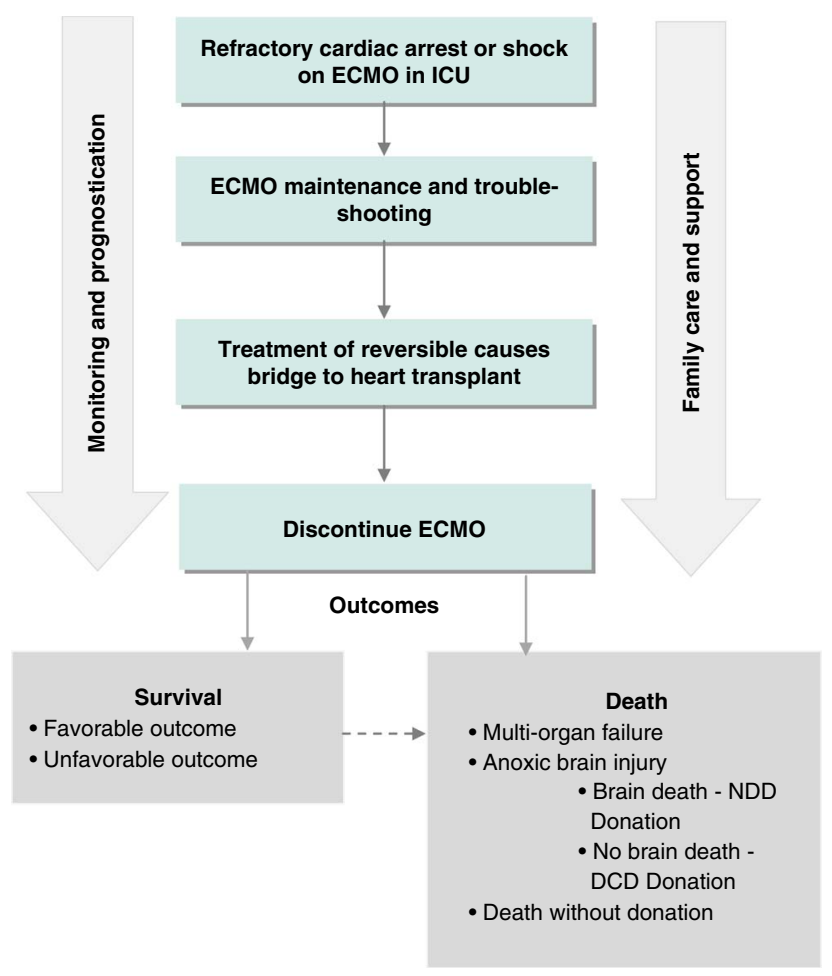

Figure 2. Clinical process frameworks for extracorporeal cardiopulmonary resuscitation in the intensive care phase of care.

$\mathrm{DCD}=$ donation after cardiac death; $\mathrm{ECMO}=$ extracorporeal membrane oxygenation; ICU = intensive care unit; $\mathrm{NDD}=$ neurological determination of death.

small group discussions, during which a representative from each group summarized the output and received input from the larger group.

The meeting closed with a plenary discussion reflecting on the outputs of the small groups and the development of a collaborative research program for ECPR to treat OHCA in the Canadian setting on the basis of knowledge gaps identified.

\section{Meeting outcomes}

\section{Summary of evidence for ECPR reviewed for the meeting}

Several moderately sized observational studies have demonstrated improved outcomes for patients treated with ECPR compared with those treated with conventional $\mathrm{CPR},{ }^{5}$ whereas some have observed no benefit associated with ECPR. ${ }^{6}$ Overall, this evidence has been rated as low quality due to methodologic limitations and a high risk of selection bias. ${ }^{5,7}$ Twenty observational studies, case series, and case reports with a total of 833 patients (ages 16 to 75 years) 
were included in a systematic review of ECPR for refractory cardiac arrest. ${ }^{8}$ Overall, $22 \%$ of patients survived to hospital discharge, including $13 \%$ who had good neurological recovery. For those studies reporting longer-term outcomes, overall survival rates were $21 \%$ at 3 months including $15 \%$ with good neurological function and $16 \%$ at 6 months including 9\% with good neurological function. Bundle treatments such as coronary revascularization, hemodynamic interventions, and targeted temperature management neuroprotection were variable between studies. The patient populations studied were variable but generally included patients ages 10 to 75 years, with a time interval from collapse to initiation of resuscitation of $<5$ to 15 minutes, a presumed cardiac etiology for their arrest, and no return of spontaneous circulation after 10 to 30 minutes. Studies generally excluded patients with a Do-Not-Resuscitate order, any disability with severe limitation of daily activities, a presumed non-cardiac cause of arrest (e.g., trauma, uncontrollable bleeding, drug overdose, poisoning, drowning, accidental hypothermia), or severe comorbidities.

Kim et al. ${ }^{9}$ performed a meta-analysis of data from 10 observational studies involving both in-hospital and OHCA to determine whether ECPR, when compared with conventional CPR, was associated with improved outcomes. ECPR was not associated with survival to hospital discharge but was associated with increased survival and good neurological outcome at 3 to 6 months.

Reviewing comparative studies published to date demonstrates significant heterogeneity in study design specifically around the population studied (e.g., variable definitions of refractory cardiac arrest), time limits for no flow and low flow states, ECPR technique (e.g., flow rates, duration of therapy), and ancillary treatments (e.g., targeted temperature management).

The 2015 Consensus on Science and Treatment Recommendations developed by the International Liaison Committee on Resuscitation (ILCOR) states, "We suggest ECPR is a reasonable rescue therapy for selected patients with cardiac arrest when initial conventional CPR is failing in settings where this can be implemented (weak recommendation, very-low-quality evidence)."

There are no randomized trials of ECPR versus conventional treatment for cardiac arrest published in the literature; however, we identified two underway at the time of the meeting. The Prague OHCA Study ${ }^{10}$ is a randomized controlled trial comparing a "hyper-invasive" treatment strategy with conventional therapy for OHCA. The hyper-invasive treatment strategy is a bundle of treatment including the use of mechanical chest compression devices by paramedics, prehospital intra-arrest cooling, and a rapid transfer of patients to the regional cardiac centre for the consideration of ECPR. The Emergency Cardiopulmonary Bypass for Cardiac Arrest study ${ }^{11}$ is a randomized control trial being conducted in Vienna. This study compares standard advanced cardiovascular life support (ACLS) treatment for OHCA with a strategy of rapid transport to an emergency department (ED) capable of ECPR.

\section{Summary of evidence provided in presentations from invited experts: ED-based ECPR programs for patients with OHCA}

Dr. Brian Grunau from the University of British Columbia outlined the ECPR program recently implemented at St. Paul's Hospital in Vancouver. Prior to starting, Dr. Grunau's group conducted research to identify the number of potential eligible patients within the catchment area and potential benefits to those patients. ${ }^{12}$ The components of the program include prehospital protocol activation; mechanical chest compression device use by emergency medical services (EMS); a rapid response ECPR team including ED staff, cardiovascular surgery, and perfusionists; emergent coronary angiography after ECPR initiation; and post-ED ECPR management in a cardiovascular intensive care unit (ICU) with ECMO expertise. Planned evaluation includes clinical and cost-effectiveness outcomes. ${ }^{13}$

Dr. Zack Shinar, an emergency physician from Sharp Memorial Hospital in San Diego, addressed meeting attendees to share his experience in implementing a pioneering ED-based ECPR program. Dr. Shinar discussed the clinical process and team organization for ECPR implementation in the ED. The roles of nurses, paramedics, and physicians were discussed, as was family support and involvement in decision-making. He reviewed their inclusion criteria for the use of ECPR (age $<70$ years, witnessed arrest, "no flow" time $<10$ minutes, reversible cause), use of diagnostic investigations and interventions for ECPR patients (e.g., cardiac catheterization), ancillary therapies such as extremity perfusion catheters, and targeted temperature management. The program has resulted in a survival rate of approximately $20 \%$ in patients with refractory cardiac arrest treated with ECPR $(n=32) .{ }^{14}$

\section{Neuroprognostication for patients treated with ECPR}

Dr. Romer Geocadin, a neuro-intensivist from Johns Hopkins provided an overview of prognostication after 
cardiac arrest. He highlighted that there is no established pre-arrest or intra-arrest factor that is a reliable predictor of neurological functional outcome. He emphasized that exit criteria for ECPR, including neuroprognostication, end-of-life decision-making, and the ability to diagnose brain death on ECMO, require further study. The process and criteria for neuroprognostication in patients after cardiac arrest, which include clinical, neurophysiologic, and imaging criteria, were reviewed. Dr. Geocadin emphasized that these parameters have not been studied adequately in the setting of ECPR. The influence of ECMO-related neurological injuries (i.e., embolic, hemorrhagic) and limitation of access to some types of neuroimaging (e.g., magnetic resonance imaging $[\mathrm{MR}])$ may also complicate the prognostic process for patients treated with ECPR.

\section{Organ donation after sudden cardiac death and ECPR}

Dr. Sam Shemie, a pediatric critical care physician and medical advisor for deceased donation to the Canadian Blood Services, presented on the intersection between ECPR and organ donation. Abdominal and thoracic organs can recover despite anoxic injury after cardiac arrest, ${ }^{15}$ including in patients treated with ECPR.
Therefore, ECPR patients may be eligible for organ donation if resuscitation efforts fail or result in severe anoxic brain injury. Existing data demonstrate that cardiac arrest during the clinical process from brain injury to donation does not impact on transplant outcomes. ${ }^{16}$ In a systematic review of international ECPR practices and outcomes, ${ }^{8}$ authors found that only 8 of 20 studies identified provided data on organ donation outcomes. A total of 88 potential deceased donors were identified among non-survivors, 17 (19\%) of whom became donors. Data on organ recipients were not reported. Dr. Shemie identified that there is an opportunity for organ donation and resuscitation communities to work together in the development of organ donation policies around the use of ECPR that are ethical and maximize opportunities for organ donation when patients treated with ECPR do not recover.

\section{Summary of knowledge gaps identified by clinical phase small groups}

High priority knowledge gaps identified in each of the three small groups defined by phases of clinical care (i.e., prehospital, ED, and critical care unit) are summarized in Boxes 1 through 3.

\footnotetext{
Box 1. Knowledge gaps identified by meeting participants related to the prehospital phase of the ECPR process. Identifying ECPR candidates in the prehospital setting

1) What are the inclusion and exclusion criteria that should be used by prehospital to identify patients appropriate for ECPR?

2) Given a set of validated or agreed upon inclusion and exclusion criteria, can paramedics reliably and accurately identify candidates for ECPR in the prehospital setting?

Prehospital ECPR

1) What is the feasibility and effectiveness of ECPR implemented in the prehospital setting as compared with no ECPR or ECPR initiated in the hospital after rapid transport for patients with OHCA? Does the feasibility or feasibility of prehospital ECPR initiation demonstrated in other countries (e.g., France, Spain) apply to the Canadian setting?

Optimizing outcomes and patient safety

1) Should mechanical chest compression devices or manual chest compressions be used for patients selected for ECPR during transport to hospital?

2) How long should resuscitation attempts be made on scene before transportation to hospital for ECPR candidates identified in the prehospital setting? Are outcomes related to the duration of resuscitation provided on scene? In other words, could patients be harmed if transported early for ECPR with suboptimal CPR compared with a strategy focusing on high-quality CPR and ACLS care on scene for a longer duration?

3) What is the risk to OHCA patients related to bypassing closer hospitals without ECPR? What factors should go into this bypass decision (Estimated time to hospital? Estimated time to cannulation? Patient factors?)

4) Should indicators of the quality of CPR delivery be routinely assessed as part of ECPR trials? What metrics of performance in conventional resuscitative techniques (e.g., chest compression fraction, proportion receiving ALS care) and systems of care (e.g., EMS response times, overall survival) should be achieved prior to the consideration of adding resource-intensive ECPR programs?

5) Is the quality of CPR provided by prehospital personnel prior to the initiation of ECPR associated with outcomes?

Provider safety

1) What are the risks to paramedics and public safety associated with rapid transport for patients selected for ECPR in the hospital?
} 
Box 2. Knowledge gaps related to the ED phase of the ECPR process identified by meeting participants.

Inclusion and exclusion criteria for ECPR

1. What are the inclusion and exclusion criteria for OHCA patients who arrive in the emergency department which identify patients most likely to benefit from ECPR? What co-morbidities are acceptable and should not be considered exclusion criteria?

2. Should patients with refractory shock post cardiac arrest be considered for ECPR on arrival to the emergency room?

3. What is the definition of "refractory" cardiac arrest? How long should conventional CPR be continued before considering ECPR?

4. Are there types of patients in which conventional CPR is less effective that could benefit from a primary strategy of ECPR as opposed to using ECPR as a salvage strategy after failed conventional advance life support measures, e.g. morbidly obese patients?

\section{ECMO readiness}

1. Where and how should ECPR equipment be stored? Is it important to keep the equipment in the emergency department to minimize delay to ECPR initiation?

2. How can the ECMO circuit be kept primed in the most efficient manner as not to waste primed circuits that expire? Should the circuits be kept primed at all times or only when receiving a pre-alert from the prehospital setting?

3. How should resuscitation rooms be set up for ECPR to ensure maximum efficiency and delivery of services?

4. What is the relative feasibility and health economics of a 24-7 ECPR program compared with a program offered during more restrictive hours (e.g. business hours)?

Personnel organization and training:

1. Who can be trained to cannulate? Can emergency physicians accomplish safe and efficient cannulation for ECPR? Should this practice be limited to other specialists like surgeons or intensivists?

2. What frequency of cannulation over time is required to maintain competency in the skill? What are the response times of these various types of cannulators and how does that impact on time to ECPR delay and outcomes?

3. Who should be the resuscitation leader when ECPR is being implemented? How best to organize the resuscitation team when ECPR is involved?

4. Who will be managing the ECMO circuit during the initial set up and maintenance in the emergency department? What is the role of the perfusionist for ECPR patients in the emergency department? Are there other professionals who could be trained to manage the ECMO circuit so that a perfusionist is not required at the bedside at all times?

5. Should all emergency department staff (nurses, physicians, allied health) be trained in ECPR or rather a subset? Does the creation of an on call ECPR team improve service delivery? What is the best method for scheduling the human resources necessary for ECPR in the emergency department? What is the effect of off-site versus on-site ECPR team members on time to ECPR initiation?

6. What is the nature of training and re-training required to maintain competency amongst ECPR team members?

\section{ETHICAL CONSIDERATIONS}

The following is a summary of the ethical issues raised and discussed during the meeting.

\section{Ensuring fair access to ECPR for OHCA}

If decisions on who receives ECPR are made on a caseby-case basis, there may be the potential for selection bias and discrimination. To avoid this, clear, evidencebased criteria for inclusion and exclusion need to be developed based on targeting those who will receive benefits. Issues of distributive justice were raised when considering the unequal access to ECMO services across geographic regions of Canada.

How should consent for ECPR be managed? Do families need to be consulted when initiating ECMO for resuscitative purposes? Is consultation with families or alternative decision-makers feasible, given the unexpected and time-sensitive nature of the intervention?

Life-saving treatments delivered to patients in emergency settings have different informed consent/family consent requirements. This is predicated on the presumption that patients would agree to the treatment if they could. Given the potential harms and uncertain outcomes with ECPR, it is not clear whether this presumption of consent could be extrapolated for ECPR.

What is the burden on patients, families, and society related to survival after ECPR with poor neurological function?

Some patients may survive after ECPR with poor neurological outcomes, including persistent coma, vegetative states, or severe disability. These catastrophic 
Box 3. Knowledge gaps for the intensive care phase of the ECPR process.

Post-arrest care

1. What are the approaches to nutrition, hemodynamic support, ventilation management, anticoagulation, sedation, and left ventricle decompression, etc., for the ECPR patient?

2. What are the indications for coronary angiography among patients treated with ECPR?

3. What is the role of targeted temperature management in patients treated with ECPR? What is the optimal target temperature management (TTM) for patients being treated with ECPR? How long should ECPR patients be treated with TTM? Does TTM during ECPR increase complication rates of ECMO (e.g. severe bleeding) or interfere with neuroprognostication?

4. What are optimal flow rates for patients treated with ECPR? How should flow rates be titrated?

Neuroprognostication and end-of-life decision-making

1. How should neuroprognostication be done for patients treated with ECPR?

a. What is the role of clinical evaluation, electrophysiology (EEG, SSEP) measures, neuroimaging, and brain blood flow and brain biomarkers with respect to neuroprognostication for patients being treated with ECPR?

b. How should confounding factors such temperature and pharmacologic agents be integrated into neuroprognostication for patients treated with ECPR?

c. What is the neuroprognostic value of having immediate return of myocardial function after ECPR initiation?

d. When should neuroprognostication happen in relation to implementation of ECPR, targeted temperature management or other therapies such as sedation to maximize accuracy for predicting outcomes?

2. In patients with return of cardiac function, are there clinical indicators (e.g. hemodynamics, neurologic function) that can identify patients suitable for safe discontinuation of ECMO? Are ECMO weaning trials necessary to optimize outcomes?

3. What are the criteria for the determination of death by neurological criteria in patients on ECMO? What are the criteria for determination of death by circulatory criteria in patients after discontinuation of ECMO?

4. For those patients with confirmed brain death or failure of cardiovascular recovery, what is the best way to manage end-of-life decisionmaking and withdrawal of ECMO in the palliative setting? This would include the development of ECMO termination rules around discontinuation of ECMO in the setting of medical futility and family refusal for withdrawal of life-sustaining therapy.

\section{Outcomes}

1. What are the most important outcomes for patients treated with ECPR and at what time points should these outcomes be measured?

2. What neurologic measure should be used to indicate the level of neurologic recovery?

3. Should quality of life tools also be standardly used?

4. Should outcomes for ECPR include organ donation and transplantation?

5. What outcomes are the most important to patients, their families and society at large?

outcomes must be considered in the overall assessment of ECPR and in the selection of outcomes in future clinical trials.

How should end-of-life management be conducted in cases where patients experience the recovery of neurological function, including awareness and alertness without the recovery of cardiac function?

In some of these patients, there may be no options for cardiac replacement therapy, including cardiac transplant or left ventricular assist devices. Ethical approaches to end-of-life decision-making and clinical management around these cases of ECPR as a "bridgeto-nowhere" must be carefully considered before implementing ECPR broadly.

How should organ donation opportunities be provided to patients treated with ECPR who do not recover?
Attendees recognized that the primary focus of any resuscitative intervention, including ECPR, must always be to save the patient's life. However, in cases where resuscitative interventions have failed, the option of organ and tissue donation should be routinely considered. Attendees recognized the need to explore ethical issues around the transition from treating patients with ECPR with a goal of survival to the use of ECMO to maintain organ viability as soon as patients are identified as nonsurvivors and potential organ donors.

\section{HEALTH ECONOMICS}

Despite a number of challenges identified, participants strongly supported the need for developing a cost-effectiveness analysis for ECPR in the setting of OHCA. Data elements specific to an economic analysis 
were considered and discussed. These included measures of clinical efficacy in life-years saved as compared to standard care, accurate accounting of direct clinical services costs associated with ECPR, the costs associated for the care of those who survive but require ongoing care due to poor neurological function, and costs and benefits resulting from organs donated by non-survivors. An ECPR program involves a series of interventions, each having different criteria for moving to the next step and each step having a variety of possible outcomes. Any evaluation of an ECPR program would need to consider unintended downstream costs and opportunity costs to the health care system. For example, use of limited resources such as perfusionist time could potentially result in cancellations of cardiac surgery, thereby increasing waiting times for cardiac surgery patients.

The group recognized that there is a lack of high quality data on many processes and outcomes related to ECPR to inform many aspects of an economic analysis. Accordingly, some aspects of the analysis would require assumptions ultimately resulting in cost-benefit estimates with wide confidence intervals. Despite these limitations, the group felt that a carefully designed cost-benefit analysis providing a range of possibilities for cost-benefit would support implementation decisions and aid in planning for this new type of clinical program being considered by many around the world.

IMPLEMENTING ECPR RESEARCH FOR PATIENTS WITH OHCA IN CANADA: OPPORTUNITIES AND CHALLENGES

\section{Implementation of ECPR clinical programs}

The general consensus among meeting participants was that ECPR for OHCA is not ready for broad implementation in Canada because of the uncertainty around clinical efficacy and cost-effectiveness. There remains a high degree of uncertainty on the optimal criteria for patient selection and many clinical aspects of the management of patients with ECPR. Nonetheless, the group recognized that some available evidence suggests feasibility and a potential to improve outcomes for selected patients when compared against conventional approaches. ${ }^{17}$ On this basis, the group was supportive of ECPR implementation for OHCA at sites with appropriate resources and training within a coordinated Canadian research framework.

Participants agreed that novel ECPR programs may be best suited for high-volume centres with well-established inpatient ECMO programs. Such centres would have a sufficient volume of eligible patients to build experience, maintain competency, and support research. ECPR for OHCA is a logistically challenging clinical activity requiring coordination across a spectrum of clinical services. ECPR is technically challenging and requires program infrastructure, institutional support, and a multidisciplinary team of experts who can participate in preparedness, team training, and continuous quality improvement.

Despite these challenges, opportunities for successful ECPR implementation in Canada were identified. With a pilot program currently underway at St. Paul's Hospital in Vancouver, Canadian experience with ECPR is growing. ${ }^{18,19}$ Attendees from across the country reported growing interest in the Canadian ECMO community regarding the use and study of ECPR, with one example being the establishment of the Canadian ECMO Interest Group (Can-ECMO). Established and developing research networks within Canada such as the Canadian Critical Care Trials Group (CCCTG), the Canadian Resuscitation Outcomes Consortium (CanROC), the Network of Canadian Emergency Researchers (NCER), the Canadian National Transplant Research Program (CNTRP), and the Canadian ECPR Research Working Group are ideal platforms to support ECPR research logistics in Canadian EDs and critical care units.

\section{A research plan for ECPR in Canada: Preparing for clinical trials involving patients with OHCA}

Participants discussed several research activities that could be initiated immediately to address identified knowledge gaps, support the implementation of ECPR clinical programs, build Canadian experience in ECPR, and inform the design and conduct of future clinical trials. Participants agreed that infrastructure funding should be sought to develop the current Canadian ECPR working group into a sustainable research consortium to plan and collaborate on ECPR research in Canada. Research activities of such a consortium would include the following:

1) Determine ECPR capacity (current and future) in Canadian healthcare institutions. The development of a national audit will measure current capacity, document current practice variability, and identify potential sites for registry participation or pilot 
programs in ECPR for OHCA. Data to gauge healthcare professional attitudes towards ECPR for OHCA, ECMO, and organ donation would enhance our understanding of barriers and facilitators to ECPR for OHCA in Canada.

2) Conduct a preliminary economic analysis on the basis of early Canadian experience in Vancouver. Participants identified a barrier to ECPR implementation with uncertain costs associated with an ECPR program to treat patients with OHCA. This information is often requested by hospital administrators and clinical leaders when ECPR programs are proposed. Using data from the St. Paul's pilot, direct and indirect costs of ECPR will be estimated. Cost-effectiveness estimates can be made by using clinical outcome data from the St. Paul's experience and extrapolation from other published reports.

3) Develop a minimum dataset for ECPR research. Building on established international standards for the reporting of clinical trials in cardiac arrest such as the Utstein criteria, ${ }^{20}$ a minimum dataset for OHCA ECPR studies should be developed. There is potential for partnering with international organizations with a stake in ECMO research and resuscitation such as the Extracorporeal Life Support Organization (ELSO) and ILCOR. Outcomes specific to organ donation need to be developed.

4) Consider the development of a Canadian OHCA ECPR registry to support observational studies. Observational studies could help determine the feasibility of ECPR in the Canadian setting and provide data to support the design of future clinical trials. Findings from observational studies could support optimization of care processes, identify patient populations who might benefit most from ECPR, and provide estimates of effect to support future clinical trial sample size calculations.

5) Develop an evidence-based consensus on inclusion/ exclusion criteria and a standardized clinical protocol for OHCA ECPR.

6) Engage with patients, families, and the public to better inform ECPR clinical practice and research.

Interviews with both survivors and families of deceased patients should be undertaken to understand short- and long-term implications of this practice from the patient and family perspectives. Public engagement was identified by attendees as a critical input to the design of any future clinical trials. Specifically, public engagement is expected to optimize the consent processes and ensure the selection of outcomes that are patient-centred. Normative claims to support the ethical issues related to the practice of ECPR should be developed with input from the public. It was recognized that the ability to provide additional support to families would be an important feature in any ECPR program. This might include family understanding and acceptance of the patient's condition, issues of consent for ECPR and research, and dealing with family conflict around end-of-life decision-making.

\section{CONCLUSION}

The first meeting of the Canadian ECPR Research Working Group was held at the $\mathrm{Li} \mathrm{Ka}$ Shing Knowledge Institute of St. Michael's in Toronto on May 4, 2016. The 32 meeting participants from across Canada included cardiac arrest researchers, an ethicist, a health economist, and clinicians from across a spectrum of specialties including paramedicine, emergency medicine, critical care, cardiac surgery, neurointensive care, ECMO, and organ donation/transplantation. The group reviewed published data and international guidelines regarding the use of ECPR, which are supportive but weak because of low quality evidence. On this basis, participants agreed that the available evidence does not support a broad implementation of ECPR for OHCA in Canada, but rather pilot implementation in select sites for the purposes of research. Participants identified several key knowledge gaps related to the use of ECPR for OHCA and identified research activities to address some of these knowledge gaps and support future Canadian clinical studies. The group agreed to pursue the development of this working group into a funded research consortium to build on, prioritize, and execute these research activities in a collaborative manner.

Acknowledgements: The members of the Canadian ECPR Research Working Group who participated in the meeting and are recognized here include Dr. Steven C. Brooks (Co-Chair), Dr. Sam Shemie (Co-Chair), Clay Gillrie, Laura Hornby, Sylvia Torrance, Jason Buick, Dr. Jim Christenson, Dr. Frederick D’Aragon, Dr. William Dick, Dr. Ian Drennan, Dr. Noam Katz, Dr. Laurie J. Morrison, Dr. Brian Grunau, Laura Hornby, Dr. Steve Lin, Dr. Dave Nagpal, Dr. Filio Billia, Dr. William 
Stansfield, Dr. Neill Adhikari, Dr. Andrew Baker, Dr. J. Gordon Boyd, Dr. Allan DeCaen, Dr. Eddy Fan, Dr. Romergryko Geocadin, Dr. John Gill, Dr. Eyal Golan, Blair Henry, and Dr. Scott Klarenbach. The participants would like to acknowledge the contribution of Dr. Zack Shiner who provided his presentation and expertise from afar.

This work was funded by Canadian Blood Services through a financial contribution from Health Canada. The views expressed herein do not necessarily represent the views of the federal, provincial, or territorial governments. Canadian Blood Services is a national, not-for-profit charitable organization that manages the supply of blood and blood products in all provinces and territories in Canada (with the exception of Quebec) and oversees the OneMatch Stem Cell and Marrow Network. Canadian Blood Services also received a mandate in 2008 for national activities related to organ and tissue donation and transplantation (OTDT), which includes development of leading practices, public awareness and education, system performance measurement, and establishment of transplant patient registries. Canadian Blood Services is not responsible for the management or funding of any Canadian organ procurement organizations (OPOs) or transplant programs. Canadian Blood Services receives its funding from the provincial and territorial Ministries of Health and the federal government, through Health Canada.

Competing interests: SCB is the principal investigator on a grant from Canadian Institutes of Health Research (CIHR) to study the PulsePoint mobile device application for out-of-hospital cardiac arrest and the principal investigator on a planning and dissemination grant from the CIHR to support the next meeting of the Canadian ECPR Research Working Group. JC is the co-principal investigator of the Canadian Resuscitation Outcomes Consortium which is co-funded by the CIHR and the Heart and Stroke Foundation of Canada. JC is also a co-investigator on the Vancouver ECPR study. LH is a paid research consultant for Canadian Blood Services and the project manager for a research program in deceased organ donation, funded by the CIHR. BG is the principal investigator of the British Columbia ECPR trial, for which Physio-Control, Inc. has provided a loan (no direct financial support) of LUCAS compression devices.

Keywords: cardiac arrest, extracorporeal cardiopulmonary resuscitation, extracorporeal membrane oxygenation

\section{SUPPLEMENTARY MATERIAL}

To view supplementary material for this article, please visit https://doi.org/10.1017/cem.2017.429

\section{REFERENCES}

1. Government Report. Extracorporeal lung support technologies - bridge to recovery and bridge to lung transplantation in adult patients: an evidence-based analysis. Ont Health Technol Assess Ser 2010;10(5):1-47.
2. Chung SY, Tong MS, Sheu JJ, et al. Short-term and longterm prognostic outcomes of patients with ST-segment elevation myocardial infarction complicated by profound cardiogenic shock undergoing early extracorporeal membrane oxygenator-assisted primary percutaneous coronary intervention. Int 7 Cardiol 2016;223:412-7.

3. Cheng A, Sun HY, Tsai MS, et al. Predictors of survival in adults undergoing extracorporeal membrane oxygenation with severe infections. 7 Thorac Cardiovasc Surg 2016; 152(6):1526-536.e1.

4. Bednarczyk JM, White CW, Ducas RA, et al. Resuscitative extracorporeal membrane oxygenation for in-hospital cardiac arrest: a Canadian observational experience. Resuscitation 2014;85(12):1713-9.

5. Callaway CW, Soar J, Aibiki M, et al. Part 4: advanced life support: 2015 International consensus on cardiopulmonary resuscitation and emergency cardiovascular care science with treatment recommendations. Circulation 2015; 132(16 Suppl 1):S84-145.

6. Choi DS, Kim T, Ro YS, et al. Extracorporeal life support and survival after out-of-hospital cardiac arrest in a nationwide registry: a propensity score-matched analysis. Resuscitation 2016;99:26-32.

7. Brooks SC, Anderson ML, Bruder E, et al. Part 6: alternative techniques and ancillary devices for cardiopulmonary resuscitation: 2015 American Heart Association guidelines update for cardiopulmonary resuscitation and emergency cardiovascular care. Circulation 2015;132(18 Suppl 2):S436-43.

8. Ortega-Deballon I, Hornby L, Shemie SD, et al. Extracorporeal resuscitation for refractory out-of-hospital cardiac arrest in adults: a systematic review of international practices and outcomes. Resuscitation 2016;101:12-20.

9. Kim SJ, Kim HJ, Lee HY, et al. Comparing extracorporeal cardiopulmonary resuscitation with conventional cardiopulmonary resuscitation: a meta-analysis. Resuscitation 2016;103:106-16.

10. Belohlavek J. Hyperinvasive approach in cardiac arrest. NCT01511666; 2016. Available at: https://clinicaltrials. gov/ct2/show/NCT01511666?term $=$ NCT01511666\&rank $=1$ (accessed October 2016).

11. Schober A. Emergency cardiopulmonary bypass for cardiac arrest (ECPB4OHCA). NCT01605409; 2016. Available at: https://clinicaltrials.gov/ct2/show/NCT01605409?term= NCT01605409\&rank=1 (accessed October 2016).

12. Grunau B, Scheuermeyer FX, Stub D, et al. Potential candidates for a structured Canadian ECPR program for out-of-hospital cardiac arrest. CFEM 2016;4:1-8.

13. Grunau B. BC ECPR trial for out-of-hospital cardiac arrest. NCT02832752; 2016. Available at: https://clinicaltrials.gov/ ct $2 /$ show/NCT02832752? term $=$ NCT02832752\& $\mathrm{rank}=1$ (accessed October 2016).

14. Bellezzo JM, Shinar Z, Davis DP, et al. Emergency physician-initiated extracorporeal cardiopulmonary resuscitation. Resuscitation 2012;83(8):966-70.

15. West S, Soar J, Callaway CW. The viability of transplanting organs from donors who underwent cardiopulmonary resuscitation: a systematic review. Resuscitation 2016;108:27-33.

16. Orioles A, Morrison WE, Rossano JW, et al. An underrecognized benefit of cardiopulmonary resuscitation: organ transplantation. Crit Care Med 2013;41(12):2794-9. 
17. Soar J, Callaway CW, Aibiki M, et al. Part 4: advanced life support: 2015 International consensus on cardiopulmonary resuscitation and emergency cardiovascular care science with treatment recommendations. Resuscitation 2015;95:e71-120.

18. Grunau B, Reynolds J, Scheuermeyer F, et al. Relationship between time-to-ROSC and survival in out-of-hospital cardiac arrest ECPR candidates: when is the best time to consider transport to hospital? Prebosp Emerg Care 2016; 20(5):615-22.

19. Grunau B, Scheuermeyer FX, Stub D, et al. Potential candidates for a structured Canadian ECPR program for out-of-hospital cardiac arrest. CFEM 2016;18(6): 453-60.
20. Perkins GD, Jacobs IG, Nadkarni VM, et al. Cardiac arrest and cardiopulmonary resuscitation outcome reports: update of the Utstein resuscitation registry templates for out-ofhospital cardiac arrest: a statement for healthcare professionals from a task force of the International Liaison Committee on resuscitation (American Heart Association, European Resuscitation Council, Australian and New Zealand Council on Resuscitation, Heart and Stroke Foundation of Canada, InterAmerican Heart Foundation, Resuscitation Council of Southern Africa, Resuscitation Council of Asia); and the American Heart Association Emergency Cardiovascular Care Committee and the Council on Cardiopulmonary, Critical Care, Perioperative and Resuscitation. Resuscitation 2015;96:328-40. 